\title{
Comparison of local tumor control in patients with HCC treated with SBRT or TACE: a propensity score analysis
}

Dominik Bettinger ${ }^{1,2^{*}}$ (D) Eleni Gkika ${ }^{3+}$, Michael Schultheiss ${ }^{1}$, Nicolas Glaser ${ }^{1}$, Sophie Lange ${ }^{1}$, Lars Maruschke ${ }^{4}$, Nico Buettner ${ }^{1}$, Simon Kirste ${ }^{3,6}$, Ursula Nestle ${ }^{3,5}$, Anca-Ligia Grosu ${ }^{3,6,7}$, Robert Thimme ${ }^{1}$ and Thomas B. Brunner $3,6,7,8$

\begin{abstract}
Background: As stereotactic body radiation therapy (SBRT) has shown to be effective and safe in patients with hepatocellular carcinoma (HCC), the aim of our propensity score matched analysis was to evaluate the efficacy of SBRT in comparison to transarterial chemoembolization (TACE) in intermediate and advanced HCC.

Methods: Patients treated with TACE $(n=367)$ and patients allocated to SBRT $(n=35)$ were enrolled in this study. Propensity score matching was performed to adjust for differences in baseline and tumor characteristics of TACE and SBRT patients. Local tumor control (LC) 1 year after treatment, overall survival (OS) and 1-year mortality were assessed.
\end{abstract}

Results: Patients treated with SBRT have received more prior HCC treatments compared to TACE patients. The LC 1 year after treatment in the unmatched cohort was $74.4 \%$ for TACE patients compared to $84.8 \%$ in the SBRT group. Patients treated with TACE showed significantly improved OS (17.0 months vs. 9.0 months, $p=0.016)$. After propensity score matching, the LC in the TACE $(n=70)$ and SBRT $(n=35)$ group was comparable $(82.9 \%$ vs. $84.8 \%, p=0.805)$ and OS did not differ significantly in both groups.

Conclusions: SBRT after prior HCC therapy in selected patients shows comparable LC at 1 year, OS and 1-year mortality compared to patients treated with TACE.

Keywords: Hepatocellular carcinoma, Transarterial chemoembolization, Stereotactic body radiation therapy, Propensity score analysis, Overall survival

\section{Background}

Hepatocellular carcinoma ( $\mathrm{HCC})$ is often diagnosed in intermediate or advanced tumor stages and treatment options are limited [1, 2]. According to the Barcelona Clinic Liver Cancer (BCLC) classification [1, 3], patients with intermediate $\mathrm{HCC}$ (BCLC B) are treated with transarterial chemoembolisation (TACE) [4] and there is growing evidence that patients with BCLC C without

* Correspondence: dominik.bettinger@uniklinik-freiburg.de

${ }^{\dagger}$ Dominik Bettinger and Eleni Gkika contributed equally to this work.

${ }^{1}$ Department of Medicine II, Medical Center University of Freiburg, Faculty of

Medicine, University of Freiburg, Hugstetter Str. 55, D-79106 Freiburg, Germany

${ }^{2}$ Berta-Ottenstein-Programme, Faculty of Medicine, University of Freiburg,

Freiburg, Germany

Full list of author information is available at the end of the article complete portal vein thrombosis (PVT) and even with extrahepatic metastases may also benefit from TACE [5].

During the last years, stereotactic body radiation therapy (SBRT) has emerged as another local ablative non-invasive treatment approach in patients with HCC [6-8]. It has been reported that SBRT can achieve high rates of local tumor control with acceptable toxicity in patients with $\mathrm{HCC}$, also in carefully selected patients with impaired liver function [6,9]. Although these reports have shown that SBRT is a feasible and well-tolerated treatment option for patients with HCC, there is no consensus in which setting SBRT should be used. SBRT was also used to bridge to liver transplantation as an alternative treatment option to TACE with favorable results [10-12]. However, there are no studies evaluating the efficacy of SBRT compared to TACE in patients with intermediate HCC outside the

(c) The Author(s). 2018 Open Access This article is distributed under the terms of the Creative Commons Attribution 4.0 International License (http://creativecommons.org/licenses/by/4.0/), which permits unrestricted use, distribution, and reproduction in any medium, provided you give appropriate credit to the original author(s) and the source, provide a link to the Creative Commons license, and indicate if changes were made. The Creative Commons Public Domain Dedication waiver (http://creativecommons.org/publicdomain/zero/1.0/) applies to the data made available in this article, unless otherwise stated. 
transplantation setting. In order to analyze this important clinical issue, we performed a single-center, retrospective analysis by using propensity score matching focusing on local tumor control, overall survival (OS) and 1-year-mortality.

\section{Methods}

\section{Selection of patients}

The TACE cohort consisted of patients who had been treated at the University Hospital Freiburg (Germany) between January 2003 and January 2015. In summary, 1030 HCC patients were included in an HCC database. Of these patients, 407 were initially treated with TACE. Patients with extrahepatic metastases who had been treated by TACE in an individual treatment approach were excluded from this analysis. Further, we excluded patients with BCLC A, who received TACE as a bridge to surgery or liver transplantation. In summary, 367 patients who have been treated with TACE were included in these analyses.

The SBRT cohort consisted of 35 consecutive patients with 49 HCC lesions who have been treated in the Department of Radiation Oncology of the University Hospital Freiburg (Germany) between 2012 and 2016 and which have partly been published elsewhere $[6,13]$. Patients treated with SBRT who received prior TACE were not included in the TACE group. Treatment decisions were made at the dedicated institutional multidisciplinary HCC tumor board following institutional, national and international guidelines. Typically, TACE was the first-line treatment in patients without complete portal vein thrombosis. SBRT was performed after TACE failure, as an alternative to systemic treatment with sorafenib or after progression during sorafenib. Therefore, these patients have mainly received prior HCC therapy.

\section{Definitions}

HCC was staged using the Barcelona Clinic Liver Cancer (BCLC) classification. Diagnosis of HCC was made according the current guidelines mainly by imaging (computer tomography $[\mathrm{CT}]$ or dynamic contrast-enhanced magnetic resonance imaging $[\mathrm{MRI}]$ ) when lesions showed the typical arterial phase hyperenhancement and portal venous and/or delayed washout $[1,3]$. The number of focal hepatic lesions and the maximum diameter as well as the presence of portal vein thrombosis (PVT) were assessed. We summarized the intrahepatic lesions in oligonodular (one or two intrahepatic lesions) and in multifocal HCC (three or more lesions or diffuse HCC growth pattern).

\section{TACE procedure}

TACE was performed using a selective or super-selective approach. Intra-arterial infusion of the chemotherapeutic agent and lipiodol was performed after having localized the target lesion. Epirubicin or mitomycin were used as chemotherapeutic agents. The chemotherapeutic agent was not defined in the study protocol. The lipiodol infusion was stopped when intra-arterial stasis was observed in the angiographic control. Further, gelatin sponge particles or PVA particles were used for embolization. In 41 patients (11.2\%) drug-eluting beads TACE (DEB-TACE) was performed.

\section{SBRT techniques}

In order to exactly define the radiation field, patients were immobilized in supine position with a vacuum cushion (BlueBAG BodyFIX, Innovative Technologies Völp, Innsbruck, Austria) and underwent 4 dimensionalCT (4D CT, Brilliance CT Big Bore, Philips Medical Systems, Cleveland, $\mathrm{OH}$ ) as previously described $[6,13]$. For the 4D acquisition (Mayo Clinic Respiratory feedback system), we monitored breathing which was reduced with an abdominal compression method. Lesions with contrast enhancement in the arterial phase and with washout in the venous phase and/or delayed phase including the portal vein thrombosis (PVT) were defined as gross tumor volume (GTV). The internal target volume (ITV) was created to account for the extent and the position of the tumor at all motion phases of the 4D-CT data set, and the PTV a uniform expansion of $4 \mathrm{~mm}$ of the ITV. Further, for using image guide radiotherapy (IGRT) lipiodol deposits from previous TACE sessions were used. In the absence of lipiodol as a marker, fiducial markers were implanted before beginning of radiotherapy. The decision for the numbers of fractions which were delivered to the patients was based on the proximity to organs at risk such as the stomach, the small intestine and the colon: In patients without a close proximity to these critical structures 3 fractions $(3 \times 12.5-15$ Gy) were preferred. In contrast, 12 fractions $(12 \times 4-5.5$ Gy) were applied if there was a close contact to the OARs and 5 fractions $(5 \times 7-$ $10 \mathrm{~Gy}$ ) were used in case of intermediate closeness to the OARs. On every treatment day, before starting radiation therapy, a cone beam computed tomography (CBCT) with oral contrast for visualizing the stomach and/or the duodenum was performed. Therefore, according to the current location of the OARs, corrections in the radiation fields were done on each treatment day if necessary.

In some lesions, dose constraints could not be achieved. In these patients, we used a simultaneous integrated protection (SIP) dose prescription without reducing the dose to the entire PVT [14]. During the study period, treatments were either prescribed to the $60 \%$ and $80 \%$ encompassing isodose (between 2007 and 2013) or according to ICRU report 83 (after 2013). The prescribed doses were converted to equieffective doses for 2 Gy fractions (EQD2) using an $\alpha / \beta$ ratio of 10 Gy and 3 Gy to account for tumour and late reacting bowel tissue, respectively. 


\section{Radiological assessment}

Radiological response was assessed every 3 months after TACE or SBRT by using the mRECIST criteria (version 1.1) [13]. Complete remission, partial remission or stable disease was summarized as local tumor control (LC). Patients treated with TACE with detection of residual HCC within the target lesion during follow-up imaging were allocated to further TACE sessions. Concerning the LC at 1 year in TACE patients we included the target lesions and reported the response assessment at 1 year. Patients who received more than one TACE session due to residual tumor disease in the target lesion were not classified as non-responders.

For response assessment in patients treated with SBRT, imaging was reviewed by comparing the treatment plan for SBRT. By using this approach, we were able to define if there was local recurrence or a new untreated tumor. The LC of SBRT patients was assessed considering all treated lesions $(n=49)$.

\section{Statistical analyses}

The present study was a retrospective observational study. Baseline characteristics of the patients were analyzed before TACE or SBRT. The primary outcome in our analysis was LC 1 year after treatment and the secondary outcome were overall survival (OS), 1-year-mortality and toxicity. Continuous variables are reported as mean with standard deviation whereas categorical variables are expressed as frequencies and percentages (in parentheses) unless stated otherwise. For continuous variables, differences were determined using Wilcoxon-Mann-Whitney and Kruskal-Wallis tests. We used non-parametric tests as there was no Gaussian distribution of the data which was confirmed by the Kolmogorov-Smirnov test before starting the analyses. $X^{2}$ tests or Fisher's Exact tests were used for categorical variables. $P$ values $<0.05$ were considered being significant.

Overall survival was defined from the day prior to TACE or SBRT until death or last follow-up. At the end of the observation period (01/07/2017) 358 patients $(89.1 \%)$ in the whole cohort and 86 patients $(81.9 \%)$ in the matched cohort had died. Survival was calculated using Kaplan-Meier analyses. Differences in survival were assessed using logRank tests.

As the outcome parameters may be influenced by patient selection for either TACE or SBRT, we performed propensity score matching. For development of the propensity score, we performed multivariable logistic regression model including the following parameters: ECOG 0 vs. $1 / 2$, segmental portal vein thrombosis (PVT), hepatic tumor expansion (oligonodular vs. multifocal), tumor size, Child score and viral liver disease. Due to the large differences of the frequency of previous treatment between the treatment groups, we were not able to adjust for this bias, as this would have resulted in very small numbers in each group after propensity score matching. After the propensity score has been established, we preformed 2:1 matching. For matching we used the nearest-neighbour matching method with a calliper with of 0.01 without replacement. Standardized differences were calculated in order to assess post-hoc balance [15]. The standardized differences before and after matching are presented in the supplementary file.

Statistical analyses were performed with SPSS (version 24.0, IBM, New York, USA) and GraphPad Prism (version 6, GraphPad Software, San Diego, CA, USA).

\section{Results}

\section{Patient characteristics}

Baseline characteristics are summarized in Table 1. In the TACE cohort there were significantly more patients with viral liver disease compared to the SBRT cohort (31.1\% vs. $11.4 \%, p=0.018)$. Patients treated with SBRT presented with more advanced tumor disease compared to patients with TACE as they were more often classified as BCLC C (18.5\% vs. $31.4 \%, p=0.046)$. $60.8 \%$ of the patients treated with TACE had multifocal HCC compared to $83.0 \%$ of the patients in the SBRT group $(p=0.010)$. Only 5 patients $(1.3 \%)$ in the TACE group had been treated before study inclusion compared to $83.0 \%$ in the SBRT group $(p<0.001)$. SBRT patients presented with a higher Child score compared to TACE patients $(5.9 \pm 1.3$ vs.8.4 \pm 7.1 , $p=0.001)$. Technical data of SBRT are summarized in Table 1.

\section{Local tumor control at 1 year, OS and 1-year mortality in patients treated with TACE or SBRT}

In patients treated with TACE the LC at 1 year was $74.4 \%$ compared to $84.8 \%$ in patients treated with SBRT $(p=0.146)$. There was a trend to a better LC in patients treated with SBRT (Table 3). Patients with TACE had a median OS of 17.0 [14.4-19.6] months compared to 9.0 [6.7-11.3] months in SBRT patients $(p=0.016)$ (Fig. 1a). 1 -year-mortality was higher in patients treated with SBRT compared to TACE patients but did not reach statistical significance ( $38.4 \%$ vs. $53.1 \%, p=0.073$, Table 3$)$.

\section{Toxicity}

The most common toxicity in patients treated with TACE was abdominal pain $(n=118,32.2 \%)$, fever $(n=84$, $22.9 \%)$ and nausea and vomiting $(n=51,14.0 \%)$. These complications developed shortly after TACE and were explained by a postembolization syndrome. Thirteen patients $(3.5 \%)$ developed hematoma after puncture of the femoral artery for angiography during the TACE procedure. Three patients $(0.8 \%)$ developed liver abscess after TACE which was treated by insertion of a percutaneous drain and antibiotic treatment. 
Table 1 Baseline characteristics of study patients and lesions treated

\begin{tabular}{|c|c|c|c|}
\hline \multirow[t]{2}{*}{ Characteristics } & TACE & SBRT & \multirow[t]{2}{*}{$p$ value } \\
\hline & $n=367$ & $n=35$ & \\
\hline Gender & & & 0.802 \\
\hline Male & $314(85.6)$ & $29(83)$ & \\
\hline Female & $53(14.4)$ & $6(17)$ & \\
\hline Age in years & $66.8 \pm 9.2$ & $69.0 \pm 8.1$ & 0.305 \\
\hline \multicolumn{4}{|l|}{$\mathrm{ECOG}^{1}$} \\
\hline 0 & $277(75.5)$ & $23(65.7)$ & 0.224 \\
\hline 1 & $43(11.7)$ & $12(34.3)$ & 0.001 \\
\hline 2 & $47(12.8)$ & 0 & 0.023 \\
\hline \multicolumn{2}{|l|}{ Etiology of liver disease } & & 0.018 \\
\hline Viral & $114(31.1)$ & $4(11.4)$ & \\
\hline Non-viral & $253(68.9)$ & 31 (88.6) & \\
\hline Child Score & $5.9 \pm 1.3$ & $6.4 \pm 1.3$ & 0.006 \\
\hline Child A & $269(73.3)$ & $19(4.3)$ & 0.020 \\
\hline Child B & $95(25.9)$ & $16(45.7)$ & 0.017 \\
\hline Child C & $3(0.8)$ & 0 & 0.999 \\
\hline Previous treatment $^{a}$ & $5(1.3)$ & $29(83.0)$ & $<0.001$ \\
\hline None & $362(98.6)$ & $6(17.1)$ & $<0.001$ \\
\hline Surgery & $2(0.5)$ & $8(22.9)$ & 0.899 \\
\hline Sorafenib & $2(0.5)$ & $1(2.9)$ & 0.324 \\
\hline TACE & $1(0.3)$ & $28(80.0)$ & $<0.001$ \\
\hline \multicolumn{2}{|l|}{$\begin{array}{l}\text { Intrahepatic tumor } \\
\text { expansion }\end{array}$} & & 0.010 \\
\hline Oligonodular & $144(39.2)$ & $6(17)$ & \\
\hline Multifocal & $223(60.8)$ & $29(83)$ & \\
\hline \multicolumn{2}{|l|}{$\mathrm{BCLC}^{2}$} & & 0.046 \\
\hline B & $299(81.5)$ & $24(68.6)$ & \\
\hline C & $68(18.5)$ & $11(31.4)$ & \\
\hline Largest tumor diameter [cm] & $6.1 \pm 3.4$ & $8.4 \pm 7.1$ & 0.001 \\
\hline Segmental $\mathrm{PVT}^{4}$ & $68(18.5)$ & $11(31.4)$ & 0.076 \\
\hline \multicolumn{4}{|l|}{ Laboratory } \\
\hline Platelets $\left[10^{3} / \mu l\right]$ & $187 \pm 115$ & $183 \pm 131$ & 0.263 \\
\hline $\mathrm{AST}^{7}[\mathrm{U} / \mathrm{I}]$ & $90 \pm 80$ & $99 \pm 66$ & 0.238 \\
\hline $\mathrm{ALT}^{8}[\mathrm{U} / \mathrm{I}]$ & $66 \pm 58$ & $55 \pm 40$ & 0.335 \\
\hline Bilirubin [mg/dl] & $1.2 \pm 1.2$ & $1.8 \pm 1.8$ & 0.656 \\
\hline Albumin $[\mathrm{g} / \mathrm{dl}]$ & $3.6 \pm 0.6$ & $3.4 \pm 0.5$ & 0.034 \\
\hline $\mathrm{AFP}^{15}[\mathrm{ng} / \mathrm{ml}]$ & $4792.4 \pm 25,171.7$ & $2279.8 \pm 9386.5$ & 0.493 \\
\hline
\end{tabular}

Technical data TACE $^{3}$ and SBRT $^{5}$

$\begin{array}{ll}\text { TACE }^{\text {CTACE }}{ }^{6} & 326(88.8) \\ \text { Drug-eluting beads TACE } & 41(11.2) \\ \text { Number of TACE sessions } & 2 \pm 1 \\ \text { Two TACE } & 253(68.9) \\ \text { Three TACE } & 84(22.9) \\ \text { Four TACE } & 30(8.2)\end{array}$

Table 1 Baseline characteristics of study patients and lesions treated (Continued)

\begin{tabular}{|c|c|c|}
\hline Characteristics & $\begin{array}{l}\text { TACE } \\
n=367\end{array}$ & $\begin{array}{l}\text { SBRT } \\
n=35\end{array}$ \\
\hline SBRT & & median $\left(\mathrm{IQR}^{14}\right)$ \\
\hline Total prescribed dose (TD) & & 45 (42-50) Gy \\
\hline $\mathrm{EQD}_{2}{ }_{10, \mathrm{TD}^{9}}{ }^{9}$ & & 56 (54-83) Gy \\
\hline $\mathrm{D}_{\max }{ }^{10}$ & & 53 (50-57) Gy \\
\hline $\mathrm{EQD2}_{10, \operatorname{Dmax}^{11}}$ & & 82 (62-98) Gy \\
\hline $\mathrm{D}_{\text {mean,liver }}{ }^{12}$ & & 17 (14-25) Gy \\
\hline EQD2 $_{\text {Dmean,liver }}{ }^{13}$ & & 20 (14-36) Gy \\
\hline
\end{tabular}

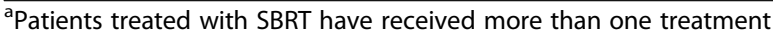
Abbreviations: ${ }^{1} E C O G$ Eastern Cooperative Oncology Group, ${ }^{2} B C L C$ Barcelona Clinic Liver Cancer ${ }^{3} T A C E$ transarterial chemoembolization, ${ }^{4} P V T$ portal vein thrombosis, ${ }^{5} S B R T$ stereotactic body radiation therapy, ${ }^{6} \mathrm{CTACE}$ conventional transarterial chemoembolization, ${ }^{7} A S T$ aspartat aminotransferase, ${ }^{8} A L T$ alanine aminotransferase, ${ }^{9} E Q D 2_{10, T D}$ equieffective doses for $2 \mathrm{~Gy}$ fractions of the prescribed dose, ${ }^{10} D_{\max }$ Maximum point dose, ${ }^{11} E Q D 2_{10, D \max }$ equieffective doses for $2 \mathrm{~Gy}$ fractions of the maximum point dose, ${ }^{12} D_{\text {mean,liver }}$ Mean liver dose, ${ }^{13} E Q D 22_{D m e a n, l i v e r}$ equieffective doses for $2 \mathrm{~Gy}$ fractions of the mean liver dose, ${ }^{14} \mathrm{IQR}$ interquartile range, ${ }^{15} \mathrm{AFP}$ alpha-fetoprotein

The adverse events of the SBRT patients included in this study have partly been published in previous studies $[6,13]$. Three of these patients developed gastric ulcer bleeding, three, four and 5 months after treatment. These patients were treated with proton pump inhibitors (2 patients, CTC grade 2$)$ and transfusion (1 patient, grade CTC 3). Importantly, the patient who developed CTC grade 3 gastroduodenitis had previously been treated with SBRT for another HCC lesion 4 months ago. Liver-associated toxicity with a deterioration of liver function assessed by an increase of the Child score was observed in 4 patients mainly with a small increase of the Child score (Child B7 to B8 and Child A6 to B7, Child A5 to A6). Only one patients showed an increase of two points of the Child score (Child A6 to B8) which was attributed to RILD. But this patient fully recovered from this deterioration of liver function and died 9 months after SBRT due to renal failure which was not attributed to treatment. The patient with an increase of the Child score from A5 to A6 after SBRT developed further hepatic decompensation without HCC progression and died 4 months after SBRT. One patient developed a necrotic abscess of the liver due to a dislocation of an indwelling Pigtail-catheter of the bile duct after stentexchange which was surgically managed and was not related to the SBRT.

\section{Propensity score matching}

As treatment allocation for TACE or SBRT is biased due to different patient and tumor characteristics, we performed propensity score matching to adjust for the imbalance concerning these factors. Multivariable logistic regression 

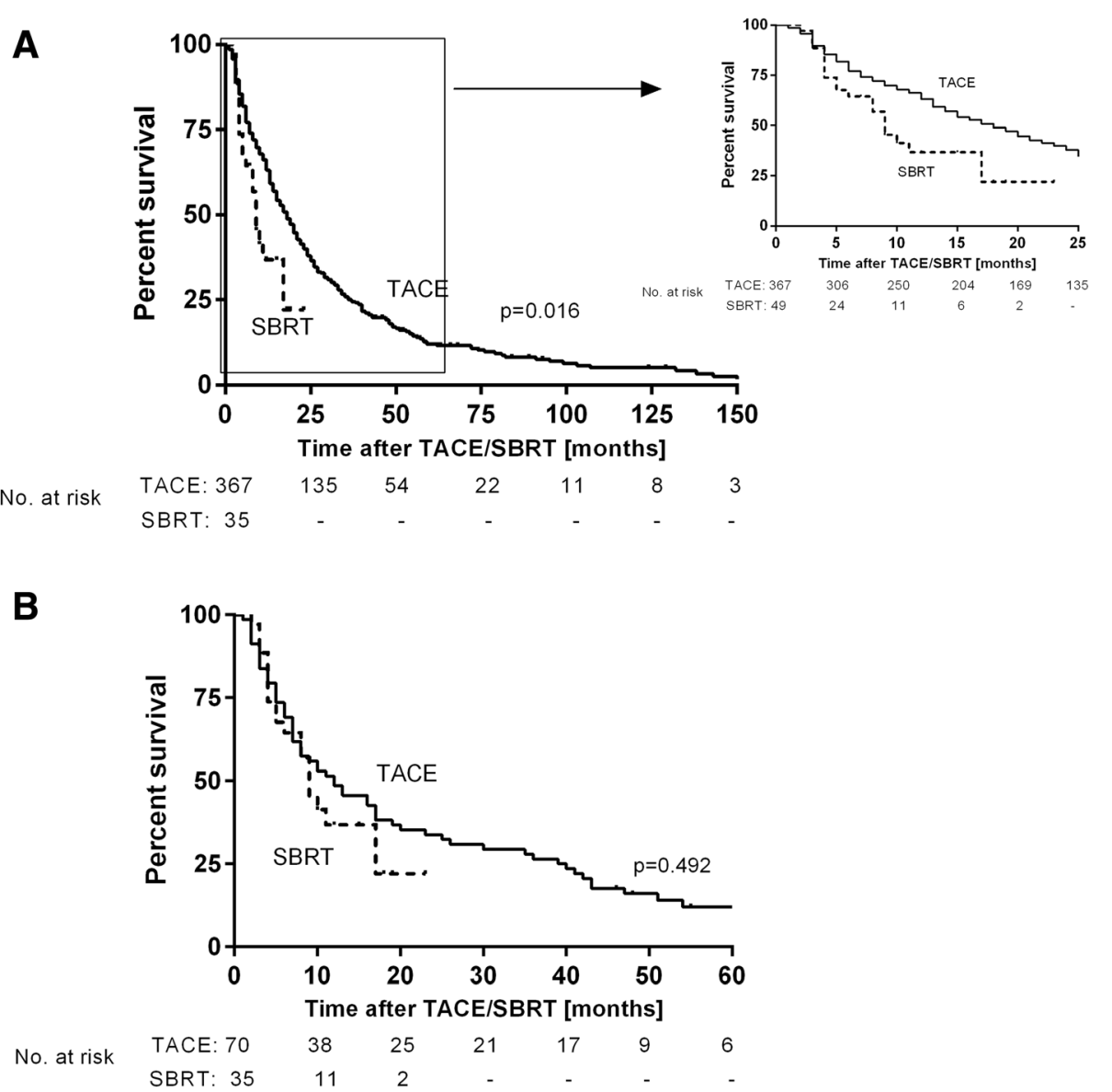

Fig. 1 Overall survival in patients with transarterial chemoembolization and SBRT in the unmatched (a) and matched cohort (b)

(Additional file 1: Table S1) analysis was performed and 105 patients after 2:1 matching (70 patients in the TACE group and 35 patients in the SBRT group) with comparable patients and tumor characteristics were identified (Table 2).

In the matched cohort, the LC at 1 year in the TACE group was $82.9 \%$ compared to $84.8 \%(p=0.805$, Table 3$)$. With regards to the OS in both cohorts, patients treated with TACE had similar OS compared to patients treated with SBRT (11.0 [5.9-16.1] months for TACE patients vs. 9.0 [6.7-11.3] months in SBRT patients, $p=0.492$, Fig. 1b). 1-year-mortality was $52.9 \%$ in the TACE cohort compared to $53.1 \%$ in the SBRT group ( $p=0.989$, Table 3).

\section{Local tumor control and 1-year-mortality in patients with $B C L C B$ and BCLC C}

We further assessed LC at 1 year and 1-year-mortality in patients in BCLC stage $B$ and $C$ (Table 3). In the matched cohort LC was comparable in BCLC B patients treated with TACE compared to SBRT patients $(83.7 \%$ vs. $82.6 \%, p=0.847)$. In patients with BCLC C LC was higher in patients by trend higher in patients treated with SBRT compared to TACE patients (87.0\% vs. $81.0 \%)$, but without reaching statistical significance $(p=0.648)$. 1-year mortality was similar in patients with BCLC B, however in BCLC C patients there was a trend to a higher 1 -year mortality in patients treated with TACE $(81.0 \%$ vs. $54.5 \%, p=0.397$, Table 3).

\section{Discussion}

SBRT is currently not included in the HCC treatment algorithm of the current European guidelines [1, 3, 16]. However, there is growing evidence that SBRT can achieve good local tumor control in patients with HCC, even in patients with advanced liver disease with acceptable toxicity $[6,17]$. Furthermore, SBRT as a bridging treatment to liver transplantation showed promising results and can be used as an alternative to conventional bridging treatments $[2,3,8,11]$. Wahl et al. showed that SBRT was equally effective compared to radiofrequency ablation [18]. Since many patients are diagnosed with intermediate or even advanced stages HCC, it is therefore important to evaluate the role of SBRT in this clinical setting. In patients with intermediate HCC, TACE is the treatment of choice [19]. Importantly, many patients are treated with several 
Table 2 Baseline characteristics of patients and treated lesions after propensity score matching

\begin{tabular}{|c|c|c|c|}
\hline \multirow[t]{2}{*}{ Characteristics } & TACE & SBRT & \multirow[t]{2}{*}{$p$ value } \\
\hline & $n=70$ & $n=35$ & \\
\hline \multicolumn{3}{|l|}{ Gender } & \multirow[t]{3}{*}{0.543} \\
\hline Male & $62(88.6)$ & $29(83.0)$ & \\
\hline Female & $8(11.4)$ & $6(17.0)$ & \\
\hline Age in years & $66.8 \pm 9.9$ & $69.0 \pm 8.1$ & 0.514 \\
\hline \multicolumn{3}{|l|}{$\mathrm{ECOG}^{1}$} & \multirow[t]{3}{*}{0.999} \\
\hline 0 & $45(64.3)$ & $23(65.7)$ & \\
\hline $1 / 2$ & $25(35.7)$ & $12(34.3)$ & \\
\hline \multicolumn{3}{|l|}{ Etiology of liver disease } & \multirow[t]{3}{*}{0.999} \\
\hline Viral & $8(11.4)$ & $4(11.4)$ & \\
\hline Non-viral & $62(86.6)$ & 31 (88.6) & \\
\hline Child Score & $6.4 \pm 1.5$ & $6.4 \pm 1.3$ & 0.952 \\
\hline Child A & $40(57.1)$ & $19(4.3)$ & 0.836 \\
\hline Child B & $30(81.4)$ & $16(45.7)$ & 0.836 \\
\hline Previous treatment ${ }^{\mathrm{a}}$ & $2(2.9)$ & $29(83.0)$ & $<0.001$ \\
\hline None & $68(97.1)$ & $6(17.1)$ & $<0.001$ \\
\hline Surgery & $1(1.4)$ & $8(22.9)$ & 0.879 \\
\hline Sorafenib & $1(1.4)$ & $1(2.9)$ & 0.001 \\
\hline TACE & 0 & $28(80.0)$ & $<0.001$ \\
\hline \multicolumn{3}{|l|}{$\begin{array}{l}\text { Intrahepatic tumor } \\
\text { expansion }\end{array}$} & \multirow[t]{3}{*}{0.999} \\
\hline Oligonodular & $13(18.6)$ & $6(17)$ & \\
\hline Multifocal & $57(81.4)$ & $29(83)$ & \\
\hline \multicolumn{3}{|l|}{$\mathrm{BCLC}^{2}$} & \multirow[t]{3}{*}{0.999} \\
\hline B & $49(70.0)$ & $24(68.6)$ & \\
\hline C & $21(30.0)$ & $11(31.4)$ & \\
\hline Largest tumor diameter $[\mathrm{cm}]$ & $8.3 \pm 4.1$ & $8.4 \pm 3.9$ & 0.845 \\
\hline Segmental $P V T^{4}$ & $21(30.0)$ & $11(31.4)$ & 0.999 \\
\hline \multicolumn{4}{|l|}{ Laboratory } \\
\hline Platelets $\left[10^{3} / \mu l\right]$ & $211 \pm 157$ & $183 \pm 131$ & 0.217 \\
\hline $\mathrm{AST}^{7}[\mathrm{U} / \mathrm{I}]$ & $98 \pm 83$ & $99 \pm 66$ & 0.742 \\
\hline $\mathrm{ALT}^{8}[\mathrm{U} / \mathrm{I}]$ & $69 \pm 61$ & $55 \pm 40$ & 0.280 \\
\hline Bilirubin [mg/dl] & $1.6 \pm 1.5$ & $1.8 \pm 1.8$ & 0.511 \\
\hline Albumin $[\mathrm{g} / \mathrm{dl}]$ & $3.6 \pm 0.7$ & $3.4 \pm 0.5$ & 0.109 \\
\hline $\operatorname{AFP}^{15}[\mathrm{ng} / \mathrm{ml}]$ & $3255.4 \pm 10,907.7$ & $2279.8 \pm 9386.5$ & 0.435 \\
\hline \multicolumn{4}{|l|}{ Technical data TACE ${ }^{3}$ and SBRT } \\
\hline \multicolumn{4}{|l|}{ TACE } \\
\hline CTACE $^{6}$ & $70(100.0)$ & & \\
\hline Drug-eluting beads TACE & 0 & & \\
\hline Number of TACE sessions & $2 \pm 1$ & & \\
\hline Two TACE & $49(70.0)$ & & \\
\hline Three TACE & $21(30.0)$ & & \\
\hline \multicolumn{2}{|l|}{ SBRT } & \multicolumn{2}{|l|}{ median $\left(\mathrm{IQR}^{14}\right)$} \\
\hline \multicolumn{2}{|l|}{ Total prescribed dose (TD) } & \multicolumn{2}{|l|}{45 (42-50) Gy } \\
\hline
\end{tabular}

Table 2 Baseline characteristics of patients and treated lesions after propensity score matching (Continued)

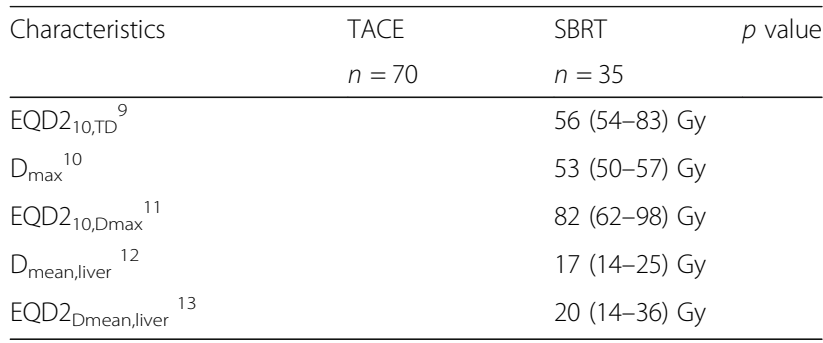

${ }^{a}$ Patients treated with SBRT have received more than one treatment Abbreviations: ' $E C O G$ Eastern Cooperative Oncology Group, ${ }^{2} B C L C$ Barcelona Clinic Liver Cancer, ${ }^{3} T A C E$ transarterial chemoembolization, ${ }^{4} \mathrm{PVT}$ portal vein thrombosis, ${ }^{5} \mathrm{SBRT}$ stereotactic body radiation therapy, ${ }^{6}$ CTACE conventional transarterial chemoembolization, ${ }^{7} A S T$ aspartat aminotransferase, ${ }^{8} A L T$ alanine aminotransferase, ${ }^{9} E Q D 2_{10, T D}$ equieffective doses for $2 \mathrm{~Gy}$ fractions of the prescribed dose, ${ }^{10} D_{\max }$ Maximum point dose, ${ }^{11} E Q D 2_{10, \text { max }}$ equieffective doses for 2 Gy fractions of the maximum point dose, ${ }^{12} D_{\text {mean,liver }}$ Mean liver dose, ${ }^{13} E Q D 2_{\text {Dmean,liver }}$ equieffective doses for 2 Gy fractions of the mean liver dose, ${ }^{14} / Q R$ interquartile range, ${ }^{15}$ AFP alpha-fetoprotein

TACE sessions to achieve a good local tumor control and in some patients further transarterial approaches may be limited due to impaired vascular architecture after several embolization procedures. In these patients sorafenib is standardly used by applying the concept of treatment stage migration. However, sorafenib is associated with several adverse events such as diarrhea and hand-foot syndrome which may limit treatment duration and therefore efficacy [20]. With regard to these adverse events which significantly reduce quality of life, SBRT may be a well-tolerated treatment [21-23]. Importantly, as shown in our unmatched cohort, patients treated with SBRT often present with advanced tumor stages. Therefore, SBRT patients had larger tumors and more often portal vein thrombosis (Table 1). In summary, there are significant differences in baseline characteristics in patients who are allocated to TACE or SBRT for HCC treatment. Being aware of these differences, we performed propensity score matching in order to adjust for these parameters which may be important for the analyzed outcome. However, as $98.6 \%$ of the patients treated with TACE had no prior HCC treatment and $83.0 \%$ of the SBRT patients had been previously been treated for HCC, we were not able to adjust for this variable as the differences were too large and sample size of the SBRT patient was too small.

After propensity score matching, we analyzed LC at 1 year after TACE or SBRT. The LC of $84.8 \%$ in SBRT patients was comparable to the LC of $82.9 \%$ in TACE patients $(p=0.805)$. Moreover, our LC at 1 year after SBRT was comparable to those reported in previous studies [6]. Further, we set out to determine the OS in our patients treated with TACE or SBRT. In the unmatched cohort, patients with TACE had significantly better OS compared to patients treated with SBRT (17.0 [14.4-19.6] 
Table 3 Summary of local tumor control and 1-year mortality in the unmatched and matched cohort in all patients and stratified in BCLC B and C

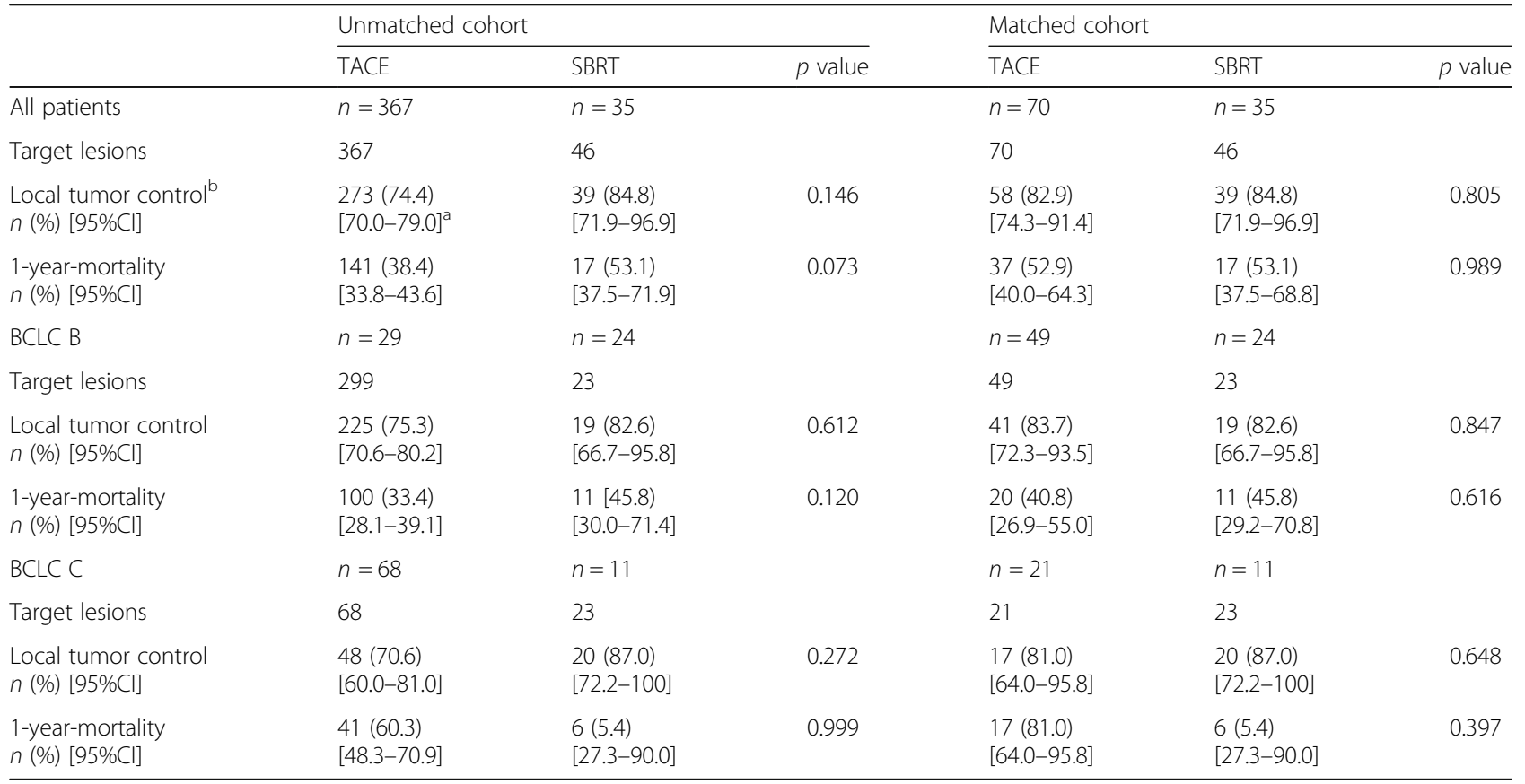

${ }^{a} 95 \% \mathrm{Cl}$ refers to the relative percentages

${ }^{b}$ Local tumor control refers to the treated target lesions

months vs. 9.0 [6.7-11.3] months, $p=0.016)$ which may be explained by the significantly different baseline characteristics as they are well-known strong prognostic factors. However, after adjusting for these confounders, OS in patients with SBRT was similar to those of patients treated with TACE (11.0 [5.9-16.1] months in TACE patients vs. 9.0 [6.7-11.3] months in SBRT patients, $p=$ 0.492). In accordance with the OS, the 1-year mortality rate in patients treated with SBRT was comparable to TACE patients $(52.9 \%$ vs. $53.1 \%, p=0.989)$. Our sub-group analyses in the matched cohort showed a trend to a higher 1-year-mortality in BCLC C patients treated with TACE compared to SBRT while LC was by trend higher in SBRT treated patients. Although not being statistically significant, these results may be the rationale for preferring TACE in BCLC B patients if technical feasible while BCLC C patients may be allocated to SBRT treatment. However, this suggestion has to be verified in prospective trials, especially taking into account prior HCC treatment, failure to previous TACE and technical feasibility of recurrent TACE.

Moreover, we evaluated adverse events after TACE and SBRT treatment. In patients treated with TACE, symptoms of postembolization syndrome occurred which resolved during symptomatic treatment. In patients treated with SBRT, although having received prior HCC treatment, toxicities were also moderate in concordance to published literature $[6,24]$. Furthermore, radiotherapy is a very well tolerated treatment in terms of quality of life with the only observed deficits being temporary worsening of appetite and fatigue [23]. Combining the good local tumor control and the few adverse events, SBRT may emerge as an effective and safe treatment in patients with intermediate HCC and also in selected patients with advanced $\mathrm{HCC}$.

We have to acknowledge several limitations of our study. Our study was a retrospective, single-center observational study with a limited sample size, especially of the SBRT patients. The decision for TACE or SBRT depended on several different factors such as intrahepatic tumor expansion, extent of PVT, liver function, the performance status of the patients and previous HCC therapies. We tried to reduce this bias by propensity score matching. However, matching was not perfect as we were not able to adjust for previous HCC therapies which would have resulted in a very small sample size without the possibility to perform statistical analyses. Therefore, prior HCC therapy may have affected outcome in patients with SBRT, especially as many of our SBRT patients had previous TACE treatment. However, according to the BCLC classification (TACE) is recommended as first-line treatment in patients with intermediate HCC. Only if TACE is technically not feasible or if contraindications do not allow to perform TACE, these patients may be allocated to SBRT treatment after multidisciplinary discussion. In summary, in everyday clinical practice, SBRT is currently not used as first-line 
treatment in these patients and therefore, these patients have received more prior HCC treatment compared to TACE patients so that this scenario represents everyday clinical practice. By considering this drawback, our results may indicate that patients who are treated with SBRT after prior HCC treatment including TACE have similar LC compared to patients who are only treated with TACE.

\section{Conclusion}

Nevertheless, our results may be the rational for designing prospective, randomized-controlled trials to analyze the efficacy of SBRT compared to TACE. With these preliminary results in mind, we have already started a prospective, single-center study comparing TACE and SBRT in this clinical setting (HERAKLES, DRKS number: DRKS00008566) in order to determine the role of SBRT in the treatment algorithm of HCC.

\section{Additional file}

Additional file 1: Table S1. Multivariate logistic regression model for propensity score matching. Figure $\mathbf{S} 1$. Standardized differences in the unmatched (black points) and matched cohort (redpoints). (DOCX 118 kb)

\begin{abstract}
Abbreviations
95\%Cl: 95\% confidence interval; AFP: Alpha-fetoprotein; ALT: Alanine aminotransferase; AST: Aspartat aminotransferase; BCLC: Barcelona Clinic Liver Cancer; BED: Biological effective doses; CBCT: Cone beam computed tomography; CT: Computerized tomography; CTACE: Conventional transarterial chemoembolization; CTC: Common toxicity criteria; Dmax: Maximum dose; EASL: European Association for The Study of Liver Diseases; ECOG: Eastern Cooperative Oncology Group; EQD2: Equieffective doses for 2 Gy fractions; GTV: Gross tumor volume; Gy: Gray; HCC: Hepatocellular carcinoma; HR: Hazard ratio; IMRT: Intensity modulated radiotherapy; IQR: Interquartile range; ITV: Internal target volume; LC: Local tumor control; MRI: Magnetic resonance imaging; NAFLD: Non-alcoholic fatty liver disease; OAR: Organ at risk; OR: Odds ratio; OS: Overall survival; PVT: Portal vein thrombosis; RILD: Radiation induced liver disease; SBRT: Stereotactic body radiation therapy; SIP: Simultaneous integrated protection; TACE: Transarterial chemoembolization; $\beta$ : Regression coefficient
\end{abstract}

\section{Funding}

The article processing charge was funded by the German Research Foundation (DFG) and the University of Freiburg in the funding programme Open Access Publishing.

DB is supported by the Berta-Ottenstein-Programme, Faculty of Medicine, University of Freiburg.

The funding body had no role in the design of the study and collection, analysis, and interpretation of data and in writing the manuscript.

\section{Availability of data and materials}

The datasets used and/or analyzed during the current study are available from the corresponding author on reasonable request.

\section{Authors' contributions}

DB, EG: study concept and design, acquisition of data, interpretation of data, statistical analyses, drafting the manuscript. MS, RT, ALG, TBB: study concept and design, acquisition of data, interpretation of data, drafting the manuscript. NG, SL, SK, UN: acquisition of data, interpretation of data, critical revision of the manuscript for important intellectual content. LM: acquisition of data, interpretation of data, critical revision of the manuscript for important intellectual content, performed transarterial chemoembolization. All authors approved the final version of the article, including the authorship.

\section{Ethics approval and consent to participate}

All patients provided written inform consent for TACE or SBRT and for data collection. This study was performed in accordance with the Declaration of Helsinki and it has been approved by the local ethics committee of the University Hospital of Freiburg (no. EK 62/14 and no. EK 350/16).

Consent for publication

Not applicable.

Competing interests

DB receives teaching and speaking fees from Bayer Healthcare.

\section{Publisher's Note}

Springer Nature remains neutral with regard to jurisdictional claims in published maps and institutional affiliations.

\section{Author details}

'Department of Medicine II, Medical Center University of Freiburg, Faculty of Medicine, University of Freiburg, Hugstetter Str. 55, D-79106 Freiburg, Germany. ${ }^{2}$ Berta-Ottenstein-Programme, Faculty of Medicine, University of Freiburg, Freiburg, Germany. ${ }^{3}$ Department of Radiation Oncology, Medical Center University of Freiburg, Faculty of Medicine, University of Freiburg, Robert-Koch-Str. 3, D-79106 Freiburg, Germany. ${ }^{4}$ Department of Radiology, Medical Center University Freiburg, Faculty of Medicine, University of Freiburg, Hugstetter Str. 55, D-79106 Freiburg, Germany. ${ }^{5}$ Department of Radiation Oncology, Kliniken Maria Hilf, Moenchengladbach, Germany. ${ }^{6}$ German Cancer Consortium (DKTK), Partner Site Freiburg, Freiburg, Germany. ${ }^{7}$ German cancer Research Center (DKFZ), Heidelberg, Germany. ${ }^{8}$ Department of Radiotherapy, University of Magdeburg, Magdeburg, Germany.

Received: 12 March 2018 Accepted: 26 July 2018

Published online: 09 August 2018

References

1. Bruix J, Sherman M. Management of hepatocellular carcinoma: an update. Hepatology. 2011;53:1020-2. https://doi.org/10.1002/hep.24199.

2. Bruix J, Gores GJ, Mazzaferro V. Hepatocellular carcinoma: clinical frontiers and perspectives. Gut. 2014;63:844-55. https://doi.org/10.1136/gutjn-2013-306627.

3. Clinical Practice Guidelines EASL. Management of hepatocellular carcinoma. J Hepatol. 2018;69:182-236. https://doi.org/10.1016/j.jhep.2018.03.019.

4. Raoul JL, Sangro B, Forner A, Mazzaferro V, Piscaglia F, Bolondi L, et al. Evolving strategies for the management of intermediate-stage hepatocellular carcinoma: available evidence and expert opinion on the use of transarterial chemoembolization. Cancer Treat Rev. 2011;37(3):212-20. https://doi.org/10.1016/j.ctrv.2010.07.006.

5. Zhao Y, Cai G, Zhou L, Liu L, Qi X, Bai M, et al. Transarterial chemoembolization in hepatocellular carcinoma with vascular invasion or extrahepatic metastasis: A systematic review. Asia Pac J Clin Oncol. 2013; 9(4):357-64. https://doi.org/10.1111/ajco.12081.

6. Gkika E, Schultheiss M, Bettinger D, Maruschke L, Neeff HP, Schulenburg M, et al. Excellent local control and tolerance profile after stereotactic body radiotherapy of advanced hepatocellular carcinoma. Radiat Oncol. 2017;12: 116. https://doi.org/10.1186/s13014-017-0851-7.

7. Qiu H, Moravan MJ, Milano MT, Usuki KY, Katz AW. SBRT for hepatocellular carcinoma: 8-year experience from a regional transplant center. J Gastrointest Cancer. 2017; https://doi.org/10.1007/s12029-017-9990-1.

8. Murray $\sqcup$, Dawson LA. Advances in stereotactic body radiation therapy for hepatocellular carcinoma. Semin Radiat Oncol. 2017;27:247-55. https://doi. org/10.1016/j.semradonc.2017.02.002.

9. Culleton S, Jiang H, Haddad CR, Kim J, Brierley J, Brade A, et al. Outcomes following definitive stereotactic body radiotherapy for patients with childPugh B or C hepatocellular carcinoma. Radiother Oncol. 2014;111:412-7. https://doi.org/10.1016/j.radonc.2014.05.002.

10. O'Connor JK, Trotter J, Davis GL, Dempster J, Klintmalm GB, Goldstein RM. Long-term outcomes of stereotactic body radiation therapy in the treatment of hepatocellular cancer as a bridge to transplantation. Liver Transplant Off Publ Am Assoc Study Liver Dis Int Liver Transplant Soc. 2012; 18:949-54. https://doi.org/10.1002/lt.23439.

11. Sapisochin G, Barry A, Doherty M, Fischer S, Goldaracena N, Rosales R, et al. Stereotactic body radiotherapy versus TACE or RFA as a bridge to transplant 
in patients with hepatocellular carcinoma An intention-to-treat analysis. J Hepatol. 2017; https://doi.org/10.1016/j.jhep.2017.02.022.

12. Katz AW, Chawla S, Qu Z, Kashyap R, Milano MT, Hezel AF. Stereotactic hypofractionated radiation therapy as a bridge to transplantation for hepatocellular carcinoma: clinical outcome and pathologic correlation. Int J Radiat Oncol Biol Phys. 2012;83:895-900. https://doi.org/10.1016/j.jijobp. 2011.08.032.

13. Bettinger D, Pinato J, Schultheiss M. Stereotactic Body Radiation Therapy as an Alternative Treatment for Patients with Hepatocellular Carcinoma Compared to Sorafenib : A Propensity Score Analysis; 2018. https://doi.org/ $10.1159 / 000490260$

14. Timmerman RD. An overview of Hypofractionation and introduction to this issue of seminars in radiation oncology. Semin Radiat Oncol. 2008;18:215-22. https://doi.org/10.1016/j.semradonc.2008.04.001.

15. Austin PC. Balance diagnostics for comparing the distribution of baseline covariates between treatment groups in propensity-score matched samples. Stat Med. 2009;28:221-39. https://doi.org/10.1002/sim.

16. Forner A, Reig M, Bruix J. Hepatocellular carcinoma. Lancet. 2018;391:130114. https://doi.org/10.1016/S0140-6736(18)30010-2.

17. Mendez Romero A, de Man RA. Stereotactic body radiation therapy for primary and metastatic liver tumors: from technological evolution to improved patient care. Best Pract Res Clin Gastroenterol. 2016;30:603-16. https://doi.org/10.1016/j.bpg.2016.06.003.

18. Wahl DR, Stenmark MH, Tao Y, Pollom EL, Caoili EM, Lawrence TS, et al. Outcomes after stereotactic body radiotherapy or radiofrequency ablation for hepatocellular carcinoma. J Clin Oncol. 2016;34:452-9. https://doi.org/10. 1200/JCO.2015.61.4925

19. Llovet JM, Bruix J. Systematic review of randomized trials for unresectable hepatocellular carcinoma: chemoembolization improves survival. Hepatology. 2003;37:429-42. https://doi.org/10.1053/jhep.2003.50047.

20. Howell J, Pinato DJ, Ramaswami R, Bettinger D, Arizumi T, Ferrari C, et al. On-target sorafenib toxicity predicts improved survival in hepatocellular carcinoma: a multi-Centre, prospective study. Aliment Pharmacol Ther. 2017; 45(8):1146-55. https://doi.org/10.1111/apt.13977.

21. Klein J, Dawson LA, Jiang H, Kim J, Dinniwell R, Brierley J, et al. Prospective longitudinal assessment of quality of life for liver Cancer patients treated with stereotactic body radiation therapy. Int J Radiat Oncol Biol Phys. 2015; 93:16-25. https://doi.org/10.1016/j.jprobp.2015.04.016.

22. Dawson LA. Overview: where does radiation therapy fit in the Spectrum of liver Cancer local-regional therapies? Semin Radiat Oncol. 2011;21:241-6. https://doi.org/10.1016/j.semradonc.2011.05.009.

23. Mendez Romero A, Wunderink W, van Os RM, Nowak PJCM, Heijmen BJM, Nuyttens JJ, et al. Quality of life after stereotactic body radiation therapy for primary and metastatic liver tumors. Int J Radiat Oncol Biol Phys. 2008;70: 1447-52. https://doi.org/10.1016/j.jprobp.2007.08.058.

24. Bujold A, Massey CA, Kim JJ, Brierley J, Cho C, RKS W, et al. Sequential Phase I and || Trials of Stereotactic Body Radiotherapy for Locally Advanced Hepatocellular Carcinoma. J Clin Oncol. 2017;31(13):1631-9. https://doi.org/ 10.1200/JCO.2012.44.1659.

Ready to submit your research? Choose BMC and benefit from:

- fast, convenient online submission

- thorough peer review by experienced researchers in your field

- rapid publication on acceptance

- support for research data, including large and complex data types

- gold Open Access which fosters wider collaboration and increased citations

- maximum visibility for your research: over $100 \mathrm{M}$ website views per year

At $\mathrm{BMC}$, research is always in progress.

Learn more biomedcentral.com/submissions 